\title{
An efficient algorithm for Padé-type approximation of the frequency response for the Helmholtz problem
}

\author{
F. Bonizzoni ${ }^{\sharp}$, F. Nobile ${ }^{\dagger}$, I. Perugia $\sharp$, D. Pradovera $^{\dagger, \star}$
}

${ }^{\sharp}$ Faculty of Mathematics, Universität Wien, Austria ${ }^{\dagger}$ CSQI, École Polytechnique Fédérale de Lausanne, Switzerland davide.pradoveradepfl.

\section{Problem setting}

Let $\Omega \subset \mathbb{R}^{d}(d=1,2,3)$ be a bounded Lipschitz domain, with boundary partitioned into the (possibly empty) sets $\Gamma_{D}$ and $\Gamma_{N}$. Given $k \in \mathbb{C}$ and $f \in L^{2}(\Omega)$, we consider the Helmholtz problem

$$
\begin{cases}-\Delta u-k^{2} u=f & \text { in } \Omega \\ u=0 & \text { on } \Gamma_{D} \\ \partial_{n} u=0 & \text { on } \Gamma_{N}\end{cases}
$$

whose solution is sought in the Hilbert space

$$
V=H_{\Gamma_{D}}^{1}(\Omega)=\left\{v \in H^{1}(\Omega),\left.v\right|_{\Gamma_{D}}=0\right\} .
$$

We can explicit the dependence on $k$ of the solution of $(\mathrm{H})$ by considering the Helmholtz solution map

$$
\begin{aligned}
\mathbb{C} & \rightarrow H_{\Gamma_{D}}^{1}(\Omega) \\
k^{2} & \mapsto \mathcal{S}\left(k^{2}\right)
\end{aligned}
$$

which associates $z=k^{2}$ to the weak solution (if it exists) of (H), i.e.

$$
\text { find } \mathcal{S}(z) \in V:(\mathcal{S}(z), v)_{H_{0}^{1}(\Omega)}-z(\mathcal{S}(z), v)_{L^{2}(\Omega)}=(f, v)_{L^{2}(\Omega)} \quad \forall v \in V .
$$

The spectral properties of the Laplacian over bounded domains imply the existence of a discrete set $\Lambda=\left\{\lambda_{\alpha}\right\}_{\alpha=1}^{\infty}$ of real values of $z$ for which (HW) is ill-posed, each associated to finite-dimensional subspace $\left\{V_{\alpha}\right\}_{\alpha=1}^{\infty} \subset V$. In particular, the subspaces $\left\{V_{\alpha}\right\}_{\alpha=1}^{\infty}$ are pairwise orthogonal and have total direct sum $V$, and

$$
\mathcal{S}(z)=\sum_{\alpha=1}^{\infty} \frac{s_{\alpha}}{\lambda_{\alpha}-z} \quad \text { with convergence in } V .
$$

Due to the self-adjointness of problem (H), the elements of $\left\{s_{\alpha}\right\}_{\alpha=1}^{\infty}$ are pairwise orthogonal, each belonging to a different eigenspace $V_{\alpha}$ of the laplacian operator.

Conclusion: $\mathcal{S}$ is meromorphic over $\mathbb{C}$, having single poles (or removable singularities) at all $\lambda \in \Lambda$. As such, we wish to approximate it using a rational function, in a Padé-like fashion. Remark: The construction of the meromorphic map $(\star)$ still holds for any problem to which the spectral theory for self-adjoint operators with compact resolvent applies.

\section{Least-squares fast Padé approximants}

Fix $z_{0} \in \mathbb{C} \backslash \Lambda$, and let $M, N, E \in \mathbb{N}$, with $E \geq \max \{M, N\}$. We define a least-squares fast Padé approximant (LSFPA) of $\mathcal{S}$ of type $[M / N]$ centered at $z_{0}$ (which depends parametrically on $E$ ) as a rational function

$$
\mathcal{S}_{[M / N]}=\mathcal{P}_{[M / N]} / \mathcal{Q}_{[M / N]}: \mathbb{C} \rightarrow V
$$

such that:

(i) $\mathcal{P}_{[M / N]} \in \mathbb{P}_{M}(\mathbb{C} ; V)=\left\{\sum_{j=0}^{M} p_{j}\left(\cdot-z_{0}\right)^{j}\right.$, with $\left.\left\{p_{j}\right\}_{j=0}^{M} \subset V\right\}$;

(ii) $\mathcal{Q}_{[M / N]} \in \mathbb{P}_{N}^{0}(\mathbb{C})=\left\{\sum_{j=0}^{N} q_{j}\left(\cdot-z_{0}\right)^{j}\right.$, with $\left.\left\{q_{j}\right\}_{j=0}^{N} \subset \mathbb{C}, \sum_{j=0}^{N}\left|q_{j}\right|^{2}=1\right\}$

(iii) $\left\|\mathcal{Q}_{[M / N]}(z) \mathcal{S}(z)-\mathcal{P}_{[M / N]}(z)\right\|_{V}=\mathcal{O}\left(\left|z-z_{0}\right|^{M+1}\right)$;

(iv) $\mathcal{Q}_{[M / N]}$ achieves the minimum over $\mathbb{P}_{N}^{0}(\mathbb{C})$ of

$$
j_{E}(Q)=\left\|(Q \mathcal{S})^{(E)}\left(z_{0}\right)\right\|_{V}=\left\|\sum_{j=0}^{N}\left(\begin{array}{c}
E \\
j
\end{array}\right) Q^{(j)}\left(z_{0}\right) \mathcal{S}^{(E-j)}\left(z_{0}\right)\right\|_{V} .
$$

\section{Theoretical results $[1]$}

Pole convergence. Let $N$ be fixed, and consider $\lambda$ among the $N$ elements of $\Lambda$ which are closest to $z_{0}$. Then there exists a root $\lambda^{\star}$ of $\mathcal{Q}_{[M / N]}$ and $C$ independent of $E$ such that

$$
\left|\lambda-\lambda^{\star}\right| \leq C\left(\frac{\rho}{R}\right)^{2 E} \quad \text { for } E \text { large enough, }
$$

with $\rho$ and $R$ being the distance from $z_{0}$ to the $N$-th and $(N+1)$-th closest elements of $\Lambda$. Remark: Numerical experiments suggest that $\rho$ may be replaced by $\left|\lambda-z_{0}\right|$.

LSFPA exponential convergence. Let $z_{0} \in \mathbb{C} \backslash \Lambda$ and $R>0$ be fixed, so that $\mathcal{B}\left(z_{0}, R\right)$ contains exactly $\bar{N}$ poles of $\mathcal{S}$, and no pole lies on $\partial \mathcal{B}\left(z_{0}, R\right)$. Consider a sequence

$$
\left(M_{k}, N_{k}\right)_{k=1}^{\infty} \subset\left\{(M, N) \in \mathbb{N}^{2}, M+1 \geq N\right\},
$$

such that $M_{k+1} \geq M_{k}$ and $N_{k+1} \geq N_{k}$ for all $k$. Moreover, assume that

$$
\infty>\lim _{k \rightarrow \infty} N_{k} \geq \bar{N} \text { and } \lim _{k \rightarrow \infty} M_{k}=\infty
$$

Let $\mathcal{S}_{\left[M_{k} / N_{k}\right]}$ be a LSFPA of $\mathcal{S}$, computed with $E \in\left\{M_{k}, M_{k}+1\right\}$ for all values of $k$. Then for any $\delta>0$, there exists $C_{\delta}$ independent of $k$ (which depends on $\lim _{k \rightarrow \infty} N_{k}$ ) such that

$$
\left\|\mathcal{S}(z)-\mathcal{S}_{\left[M_{k} / N_{k}\right]}(z)\right\|_{V} \leq C_{\delta}\left(\frac{\left|z-z_{0}\right|}{R}\right)^{M_{k}+1} \quad \text { for } k \text { large enough, }
$$

for all $z \in \mathcal{B}\left(z_{0}, R\right) \backslash \mathcal{E}_{\delta}$, with $\left|\mathcal{E}_{\delta}\right|<\delta$

LSFPA convergence in measure. Take the hypotheses above except for the boundedness assumption on the sequence $\left(N_{k}\right)_{k=1}^{\infty}$. There exists a set of zero measure $\mathcal{E}_{0}$ such that

$$
\lim _{k \rightarrow \infty}\left\|\mathcal{S}(z)-\mathcal{S}_{\left[M_{k} / N_{k}\right]}(z)\right\|_{V}=0
$$

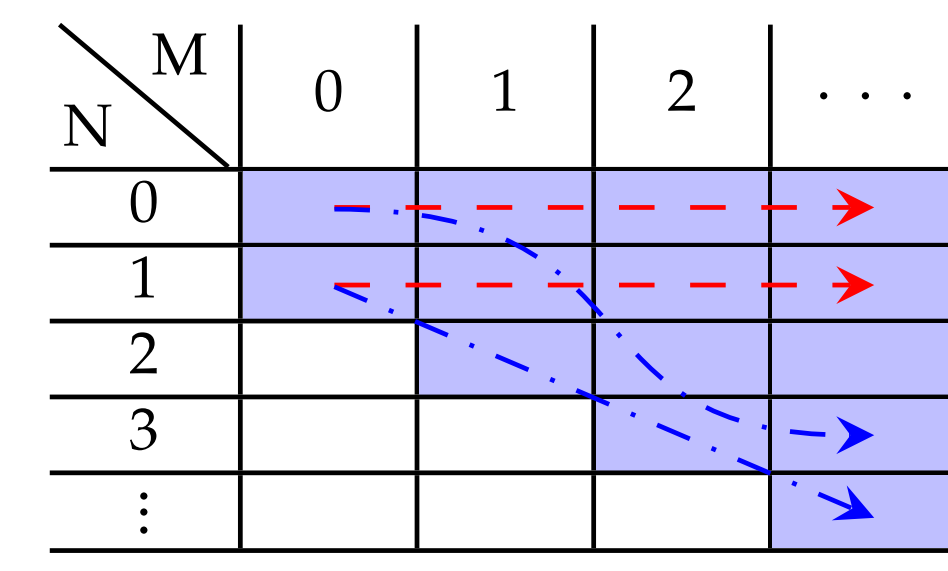

Figure 1: Padé table. In blue the region of convergence.

for all $z \in \mathcal{B}\left(z_{0}, R\right) \backslash \mathcal{E}_{0}$

ntial rate along rows of the Pade table, and with no specific rate on more general paths along the Padé table.

table, and with no specific rate on more general paths along the Pade table. Remark: More refined estimates on the general convergence rate of LSFPAs can be obtained

\section{Implementation-oriented remarks}

The numerical computation of LSFPAs relies on three steps:

- Evaluation of derivatives. The derivatives of the Helmholtz map are computed up to degree $E$, according to the recursive definition (which follows from (HW))

find $\mathcal{S}^{(j)}\left(z_{0}\right) \in V$

$$
\left(\mathcal{S}^{(j)}\left(z_{0}\right), v\right)_{H_{0}^{1}(\Omega)}-z_{0}\left(\mathcal{S}^{(j)}\left(z_{0}\right), v\right)_{L^{2}(\Omega)}=j\left(\mathcal{S}^{(j-1)}\left(z_{0}\right), v\right)_{L^{2}(\Omega)} \quad \forall v \in V,
$$

for $j=1, \ldots, E$, with the base case being $\mathcal{S}^{(0)}\left(z_{0}\right)=\mathcal{S}\left(z_{0}\right)$.

Remark: The span of the derivatives of $\mathcal{S}$ is a Krylov subspace of $V$ generated by the resolvent at $z_{0}\left(-\Delta-z_{0}\right)^{-1}: H^{-1}(\Omega) \rightarrow H_{\Gamma_{D}}^{1}(\Omega)$. Hence, the sequence of problems above can be solved efficiently by computing and storing a single operator factorization.

- Optimizing. The minimization of (F) can be recast as a constrained quadratic programming problem, effectively coinciding with the computation of the minimal eigenpair of a $(N+1) \times(N+1)$ Hermitian matrix. The components of the eigenvector provide the coefficients of the LSFPA denominator.

- Assembling. Once the denominator has been computed, the (unique) numerator satisfying (iii) can be found by matching the first Taylor coefficients (at $z_{0}$ ) of $\mathcal{P}_{[M / N]}$ with those of $\mathcal{Q}_{[M / N]} \mathcal{S}$

\section{Numerical example: damaged elastic membrane}

We consider a membrane $\Omega=[0,2] \times[0,1] \backslash T$, with $T$ being a thin triangular "crack" in the upper side of the rectangle, see figure below. We consider a time-periodic Gaussian load

$$
f^{\star}(\boldsymbol{x}, t)=\operatorname{Re}\left(\exp \left\{i k t-\frac{\left|\boldsymbol{x}-\boldsymbol{x}_{0}\right|^{2}}{2 \sigma^{2}}\right\}\right)
$$

centered at $\boldsymbol{x}_{0}=\left(\frac{1}{2}, \frac{1}{2}\right)^{\top}$ and with standard deviation $\sigma=\frac{1}{10}$. We assume that the membrane is held in place over all its border, except on the stress-free crack.

We seek the solution $u=\mathcal{S}(z)$ of the Helmholtz equation $(\mathrm{H})$ with $f(\boldsymbol{x})=f^{\star}(\boldsymbol{x}, 0)$, which represents the asymptotic amplitude of a monocrome (with wavenumber $k$ ) solution of the wave equation (with normalized wave speed) whose forcing term is $f^{\star}[4]$.

In order to approximate the solution map $\mathcal{S}$ for several values of $k^{2}=z \in[78,122]$, we apply LSFPAs with center at $z_{0}=100+\frac{1}{2} i$ and several values of $M, N$ and $E$. In particular, we do not approximate the solution of $(\mathrm{H})$ - or more properly $(\mathrm{HW})$ - directly, but we consider a discretization of the problem with $\mathbb{P}^{3}$ Finite Elements on a triangular mesh
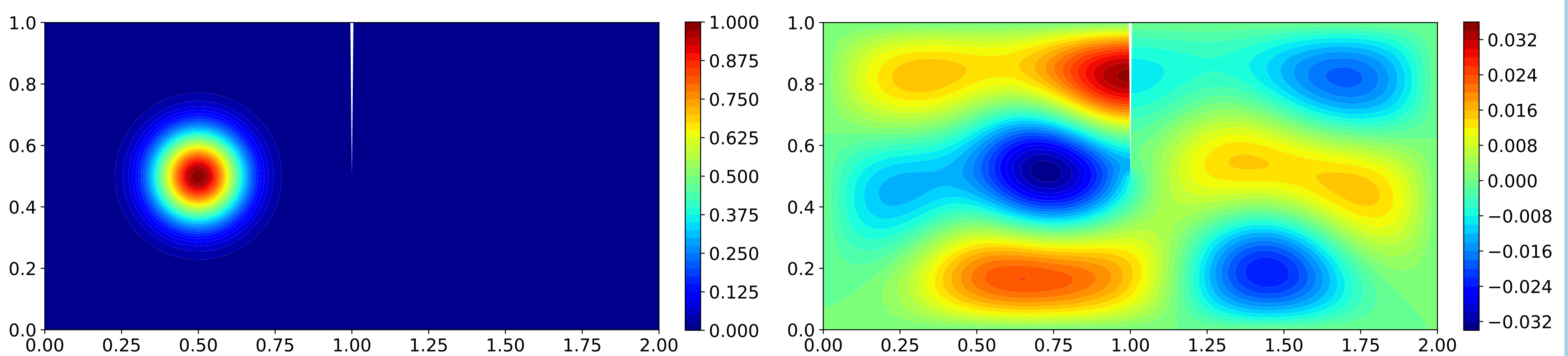

Figure 2: Amplitude $f$ of the load term (left) and solution of $(\mathrm{H})$ for $k^{2}=z=100$ (right).
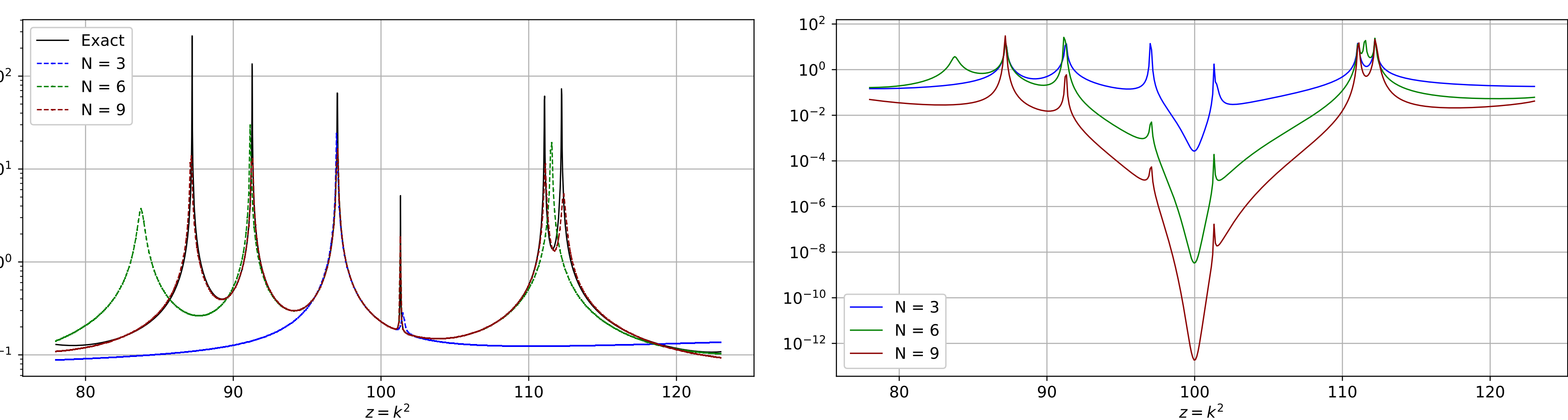

Figure 3: $V$-norm of $\mathcal{S}$ and of $\mathcal{S}_{[M / N]}$ (left) and of the approximation error (right) for sub-diagonal approximants of type $[N-1 / N]$ for $N \in\{3,6,9\}$, with $z \in[78,122]$. In all cases, $E$ is equal to $N$.

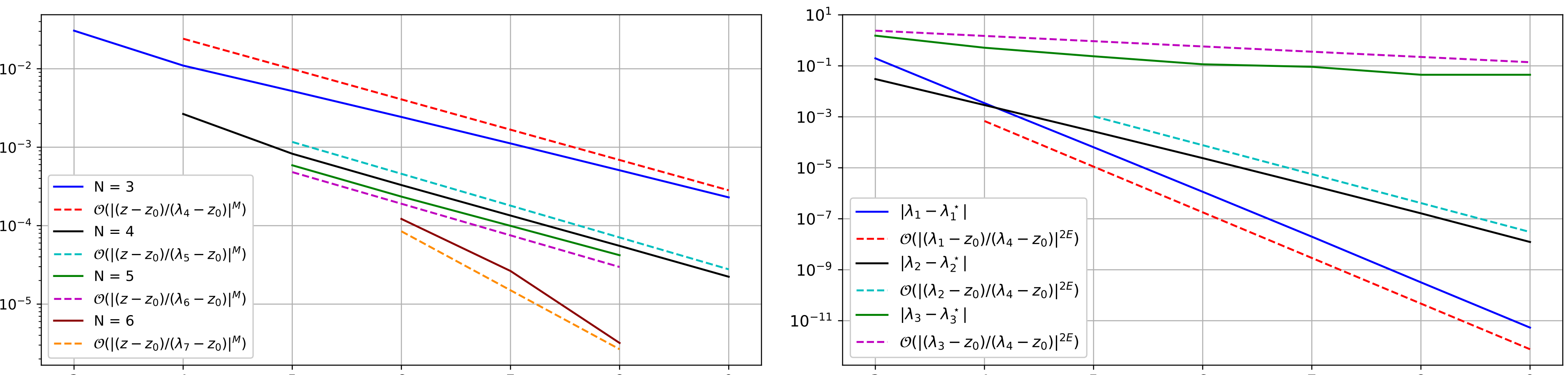

Figure 4: Convergence of the approximation error Figure 5: Convergence of the roots of LSFPAs dein the $V$-norm at $z=105$ for several choices of nominators for $N=3$ to the $N$ poles of $\mathcal{S}$ which $N$. In all cases, $E$ is set equal to $M$. are closest to $z_{0}$

Conclusion: On rows of the Padé table, i.e. for fixed $N$, the theoretical convergence rates for both the approximation error and the distance between exact and estimated poles have been verified. Moreover, the convergence along the sub-diagonal sequence $([N-1 / N])_{N=1}^{\infty}$ has been confirmed numerically.

Remark: The $V$-norm used in the experiments above is a suitably weighted version

$$
\|\cdot\|_{V}=\left(\|\cdot\|_{H_{0}^{1}(\Omega)}^{2}+\operatorname{Re}\left(z_{0}\right)^{2}\|\cdot\|_{L^{2}(\Omega)}^{2}\right)^{1 / 2}
$$

of the usual $H^{1}(\Omega)$ norm to account for the harmonic nature of the solution.

\section{References}

[1] F. Bonizzoni, F. Nobile, I. Perugia, D. Pradovera, Fast least-squares Padé approximation for self-adjoint problems with meromorphic structure, in preparation.

[2] F. Bonizzoni, F. Nobile, and I. Perugia. Convergence analysis of Padé approximations for Helmholtz frequency response problems, Mathematical Modelling and Numerical Analysis, 2017.

[3] F. Bonizzoni, F. Nobile, I. Perugia, D. Pradovera, Padé approximation of parametric and stochastic Helmholtz maps, in preparation.

[4] P. Monk, Finite Element methods for Maxwell's equations, Oxford Clarendon, 2003. 